\title{
Efficacy of gemcitabine combined with oxaliplatin, L-asparaginase and dexamethasone in patients with newly-diagnosed extranodal NK/T-cell lymphoma
}

\author{
HONG-QIANG GUO ${ }^{1,2^{*}}$, LEI LIU ${ }^{1,2 *}$, XIN-FENG WANG ${ }^{1,2}$, TONG-YU LIN $^{3}$, \\ SHU-NA YAO ${ }^{1,2}$, ZHI-HUA YAO ${ }^{1,2}$ and SHU-JUN YANG ${ }^{1,2}$ \\ ${ }^{1}$ Department of Medical Oncology, The Affiliated Cancer Hospital of Zhengzhou University; ${ }^{2}$ Department of Medical \\ Oncology, Henan Province Cancer Hospital, Zhengzhou, Henan 450008; ${ }^{3}$ Department of Medical Oncology, \\ Cancer Center, Sun Yat-sen University, Guangzhou, Guangdong 510060, P.R. China
}

Received May 23, 2014; Accepted July 21, 2014

DOI: $10.3892 / \mathrm{mco} .2014 .368$

\begin{abstract}
There is currently no standard first-line regimen for patients with extranodal natural killer (NK)/T-cell lymphoma (ENKTCL). In this study, we investigated the efficacy and toxicity of gemcitabine (GEM) combined with oxaliplatin (L-OHP), L-asparaginase (L-ASP) and dexamethasone (DXM) (GOLD regimen) as a systemic treatment scheme for newly-diagnosed ENKTCL cases. A total of 55 patients were recruited at the Henan Province Cancer Hospital and the Cancer Center of Sun Yat-sen University between May, 2008 and August, 2012. The GOLD regimen included a 14-day treatment cycle with GEM $\left(1,000 \mathrm{mg} / \mathrm{m}^{2}\right)$ on day 1 , L-OHP $\left(100 \mathrm{mg} / \mathrm{m}^{2}\right)$ on day 1 , L-ASP $\left(10,000 \mathrm{U} / \mathrm{m}^{2}\right)$ on days $1-5$ and DXM (20 mg b.i.d.) on days 1-4. The response rate, survival rate and treatment toxicity were analyzed. The overall response rate was $91 \%(48 / 55)$ with a complete response in $62 \%(34 / 55)$ and a partial response in $29 \%(15 / 55)$ of the patients. For all patients, the 1-, 2- and 3-year progression-free survival (PFS)
\end{abstract}

Correspondence to: Dr Tong-Yu Lin, Department of Medical Oncology, Cancer Center, Sun Yat-sen University, 651 DongFeng Road, Guangzhou, Guangdong 510060, P.R. China

E-mail: tongyulin@hotmail.com

Dr Hong-Qiang Guo, Department of Medical Oncology, The Affiliated Cancer Hospital of Zhengzhou University, 127 DongMing Road, Zhengzhou, Henan 450008, P.R. China

E-mail: guohq365@aliyun.com

*Contributed equally

Abbreviations: ENKTCL, extranodal NK/T-cell lymphoma; GEM, gemcitabine; L-OHP, oxaliplatin; L-ASP, L-asparaginase; DXM, dexamethasone; LDH, lactate dehydrogenase; ECOG PS, Eastern Cooperative Oncology Group performance status; HPS, hemophagocytic syndrome; IFRT, involved-field radiation therapy; LMWH, low-molecular weight heparin

Key words: extranodal natural killer/T-cell lymphoma, chemotherapy, GOLD regimen, efficacy, toxicity rate was 86,64 and $57 \%$ and the overall survival (OS) 91, 80 and $74 \%$, respectively. The 1-year PFS in patients with stage I/II vs. those with III/IV disease was 87 vs. $66 \%(\mathrm{P}<0.001)$ and the 1-year OS was 98 vs. $75 \%$, respectively $(\mathrm{P}<0.001)$. No chemotherapy-related mortality or severe complications were recorded. In conclusion, the GOLD regimen was found to be highly effective and safe for the treatment of patients with newly-diagnosed ENKTCL.

\section{Introduction}

In the 2008 World Health Organization (WHO) classification system for malignant lymphoma, extranodal natural killer (NK)/T-cell lymphomas (ENKTCL) are considered to be a distinct entity. ENKTCLs are significantly more common in Asia and Latin America compared to Europe and North America $(1,2)$. We collected a total of 4,801 cases with lymphoma in South China and 963 cases fulfilled the diagnostic criteria of mature T-cell or NK/T-cell lymphoma and accounted for $20.1 \%$ of all cases of lymphoma encountered during the same period. Of these cases, 281 (29.2\%) were diagnosed as ENKTCL, nasal type (3). Radiotherapy was the treatment selection for early-stage nasal ENKTCL. However, distant and local relapse often occurred and the 5-year overall survival (OS) was low (29.8-66\%) (4-7). An optimal treatment for ENKTCL has not yet been established, particularly for advanced-stage disease. The low treatment efficacy may be mainly attributed to the fact that this disease is resistant to several chemotherapeutic agents, due to the expression of P-glycoprotein (P-gp) $(8,9)$. Therefore, investigations have been focused on improving the efficacy of chemotherapy and reducing the risk of disease recurrence. It has been reported that L-asparaginase (L-ASP) and gemcitabine (GEM) may achieve satisfactory response and survival rates, as they are not regulated by P-gp in relapsed and refractory ENKTCL (10-14). GEM combined with oxaliplatin (L-OHP) has also been verified to be an effective salvage regimen for patients with relapsed or refractory B-cell lymphoma, with acceptable toxicity (15-17). Nowak-Göttl et al (18) reported that the use of dexamethasone (DXM) instead of 
prednisone significantly reduced the incidence of venous thromboembolism, which is a common adverse reaction of L-ASP. Therefore, we investigated the efficacy and toxicity of GEM combined with L-OHP, L-ASP and DXM (GOLD regimen) in patients with newly-diagnosed ENKTCL.

\section{Patients and methods}

Patient eligibility. A total of 55 patients were recruited at the Henan Province Cancer Hospital and the Cancer Center of Sun Yat-sen University between May, 2008 and August, 2012. Only patients aged $>15$ years were considered to be eligible for this study. Additional eligibility criteria included ENKTCL histologically confirmed by biopsy, with measurable lesions radiologically; normal hepatic and renal function; and adequate bone marrow reserve without previous treatment. Patients were excluded if they had severe organ dysfunction or concomitant malignant tumors. The diagnosis was established according to the WHO classification criteria. Aggressive NK/T-cell leukemia was excluded. The lesions were staged according to the Ann Arbor staging system. Additional examinations included complete blood count, serum biochemistry and lactate dehydrogenase (LDH) levels (19); in addition, the Eastern Cooperative Oncology Group performance status (ECOG PS) and presence of hemophagocytic syndrome (HPS) and/or B symptoms were assessed. All the patients underwent bone marrow aspiration and biopsy, computed tomographic scanning of the involved organ(s), chest and abdomen and nasal endoscopic examination.

Informed consent was obtained from all the subjects and the study was approved by the Medical Ethics Committee of the Henan Province Cancer Hospital.

Chemotherapeutic regimen. This treatment scheme was a 14-day cycle and included four drugs, namely GEM, L-OHP, L-ASP and DXM. The patients received GEM $\left(1,000 \mathrm{mg} / \mathrm{m}^{2}\right)$ on day 1 , L-OHP $\left(100 \mathrm{mg} / \mathrm{m}^{2}\right)$ on day 1 , L-ASP (Changzhou Qianhong Bio-pharma Co., Ltd, Changzhou, China) $\left(10,000 \mathrm{U} / \mathrm{m}^{2}\right)$ on days $1-5$ and DXM (20 mg b.i.d.) on days 1-4. An intradermal test was required prior to the administration of L-ASP. Every patient received chemotherapy for $\geq 4$ cycles.

All the patients with Ann Arbor stage I/II disease received involved-field radiation (IFRT) following chemotherapy. The decision was made at the discretion of the treating physician. Three-dimensional conformal radiotherapy was performed with a linear accelerator at a daily fraction of $2.0 \mathrm{~Gy}$. The total dose was $\geq 50$ Gy.

Response to treatment and toxicity assessment. Tumor response was classified as complete response (CR), partial response (PR), stable disease (SD), or progressive disease (PD), according to the report of an International Workshop to standardize the response criteria for non-Hodgkin's lymphomas. All the adverse reactions were evaluated according to the National Cancer Institute Common Toxicity Criteria, version $3(20,21)$.

Statistical analysis. OS was calculated from the date of treatment initiation to the date of the last follow-up or death from any cause. Progression-free survival (PFS) was measured
Table I. Patient characteristics $(n=55)$.

\begin{tabular}{|c|c|c|}
\hline Characteristics & Patient no. & Percentage \\
\hline \multicolumn{3}{|l|}{ Gender } \\
\hline Male & 41 & 75 \\
\hline Female & 14 & 25 \\
\hline \multicolumn{3}{|l|}{ Age (years) } \\
\hline $14-20$ & 6 & 10 \\
\hline $21-60$ & 41 & 75 \\
\hline $60-80$ & 8 & 15 \\
\hline \multicolumn{3}{|l|}{ ECOG PS } \\
\hline 0 & 42 & 76 \\
\hline 1 & 9 & 16 \\
\hline 2 & 4 & 8 \\
\hline \multicolumn{3}{|l|}{ B symptoms } \\
\hline Yes & 32 & 58 \\
\hline No & 23 & 42 \\
\hline \multicolumn{3}{|l|}{ Ann Arbor stage } \\
\hline I/II & 45 & 82 \\
\hline III/IV & 10 & 18 \\
\hline \multicolumn{3}{|l|}{ IPI } \\
\hline 1 & 29 & 53 \\
\hline 2 & 16 & 29 \\
\hline 3 & 8 & 15 \\
\hline 4 & 2 & 3 \\
\hline \multicolumn{3}{|l|}{ LDH level } \\
\hline Elevated & 12 & 22 \\
\hline Normal & 43 & 78 \\
\hline \multicolumn{3}{|l|}{ HPS } \\
\hline Yes & 10 & 18 \\
\hline No & 45 & 82 \\
\hline \multicolumn{3}{|l|}{ Involved sites } \\
\hline Nasal cavity/nasopharynx & 48 & 87 \\
\hline Other & 7 & 13 \\
\hline
\end{tabular}

ECOG PS, Eastern Cooperative Oncology Group performance status; IPI, international prognostic index; LDH, lactate dehydrogenase; HPS, hemophagocytic syndrome.

Table II. Response rate after the GOLD regimen.

\begin{tabular}{lcc}
\hline Type of response & Patient no. & Percentage \\
\hline Complete response & 34 & 62 \\
Partial response & 16 & 29 \\
Stable disease & 2 & 4 \\
Progressive disease & 3 & 5 \\
\hline
\end{tabular}

from the date of diagnosis to relapse, death or last follow-up visit. Survival was estimated using Kaplan-Meier curves and compared by the log-rank test. $\mathrm{P}<0.05$ with two-side test was considered to indicate a statistically significant difference. 

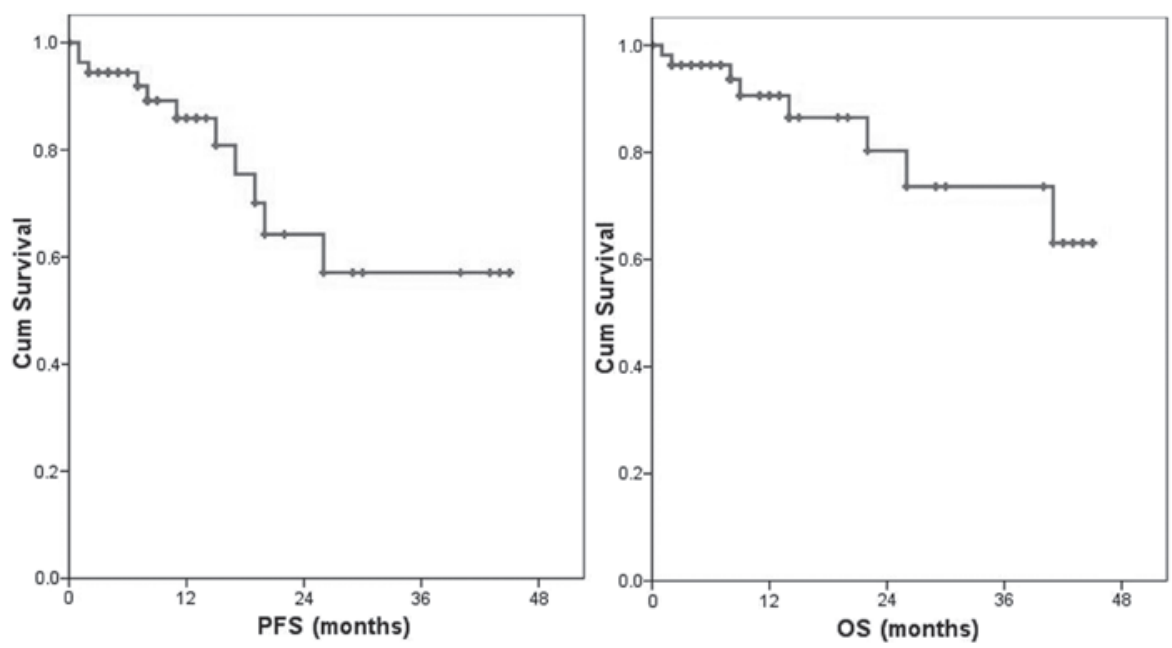

Figure 1. Progression-free survival (PFS) and overall survival (OS) of all patients. The 1-, 2- and 3-year PFS rate was 86,64 and $57 \%$ and the OS rate was 91 , 80 and $74 \%$. Cum, cumulative.
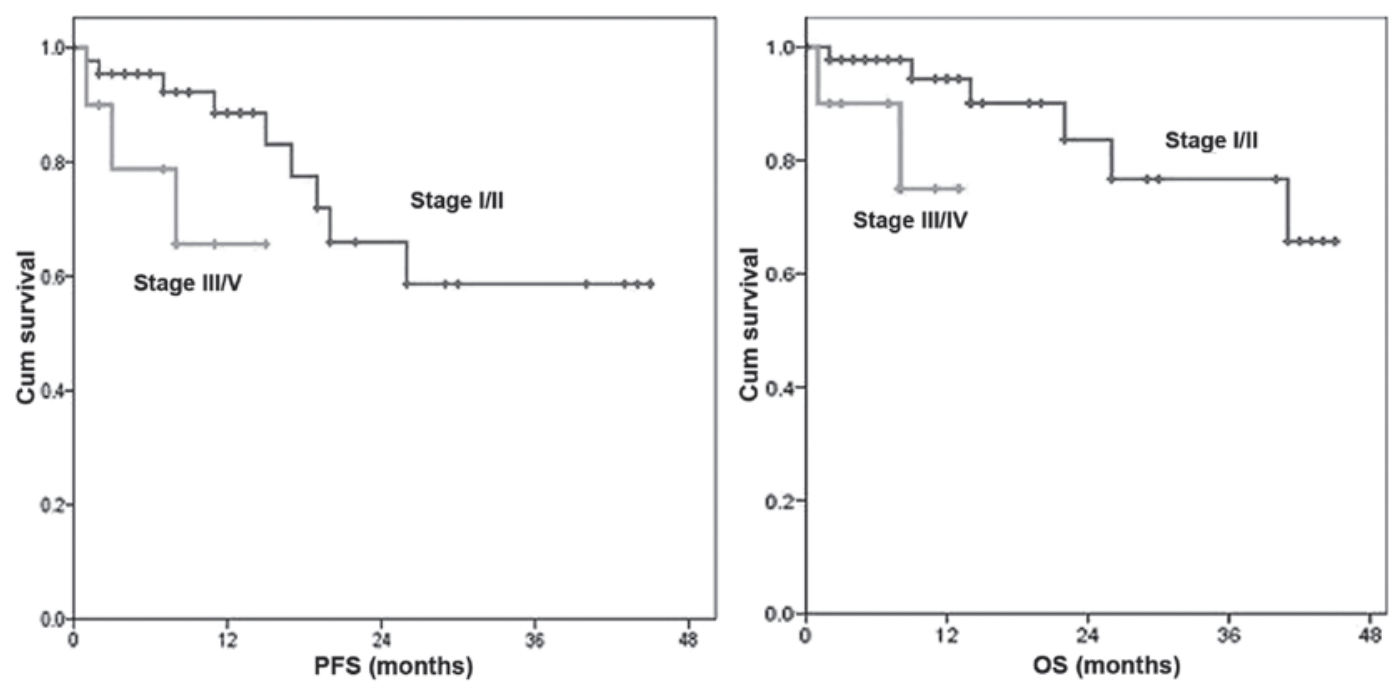

Figure 2. Comparison of progression-free survival (PFS) and overall survival (OS) between patients with stage I/II and III/IV disease. The 1-year PFS in patients with stage I/II vs. those with III/IV disease was 87 vs. $66 \%(\mathrm{P}<0.001)$ and the 1 -year OS was 98 vs. $75 \%$, respectively $(\mathrm{P}<0.001)$. Cum, cumulative.

\section{Results}

Patient characteristics. A total of 55 patients were recruited from two centers. The median age was 41 years (range, 15-69 years) and the patients were mainly young adults. The male:female ratio was $3: 1$. The majority of the patients had a good ECOG PS and international prognostic index and early-stage disease by the Ann Arbor staging system. B symptoms were present in $58 \%$ of the patients at diagnosis and HPS occurred in $18 \%$ patients. The majority of the lesions $(87 \%)$ were located in the nasal cavity or nasopharynx (Table I).

Response to treatment and survival outcomes. A total of 48 patients $(91 \%)$ responded to the GOLD regimen, with 34 cases (62\%) achieving CR and 16 (29\%) a PR, whereas 2 patients achieved SD and 3 developed PD (Table II). Of the patients with $\mathrm{CR}, 25$ received radiotherapy sequentially. Of the patients with PR, 10 were treated with radiotherapy, of whom
6 achieved a CR, 3 a PR and 1 exhibited PD. Two patients with $\mathrm{SD}$ received radiotherapy, with one patient achieving a $\mathrm{CR}$ and the other SD. Three patients with PD refused the second-line treatment.

For all patients, the 1-, 2- and 3-year PFS was 86, 64 and $57 \%$ and the OS was 91,80 and $74 \%$, respectively (Fig. 1). Eleven patients were relapse. Eight patients presented with systemic recurrence and 3 patients with local recurrence. The 1 -year PFS in patients with stage I/II vs. those with III/IV disease was 87 vs. $66 \%(\mathrm{P}<0.001)$ and the 1 -year OS was 98 vs. $75 \%$, respectively $(\mathrm{P}<0.001)$ (Fig. 2).

Toxicity. In our study, myelosuppression was observed in 16 patients, with grade $1 / 2$ neutropenia and/or thrombocytopenia in 5 cases, grade $3 / 4$ neutropenia and/or thrombocytopenia in 9 cases and febrile neutropenia in 2 cases. Hepatic dysfunction was observed in 2 patients. The levels of aminotransferases were $>200 \mathrm{U} / 1$ and returned to normal 
following treatment with liver protectants. Mild coagulation abnormalities were observed in 19 patients, reflected by a prolongation of the activated partial thromboplastin time and D-dimer elevation. One patient with elevated D-dimers and decreased fibrinogen levels prior to treatment returned to normal levels after chemotherapy and treatment with low-molecular weight heparin (LMWH). Another patient developed left lower limb deep venous thromboembolism and revascularization was achieved following treatment with urokinase and LMWH. The consumption of fatty foods was prohibited to lower the risk of pancreatitis and no such events were reported. All the patients exhibited some peripheral nervous system toxicity and grade $1 / 2$ gastrointestinal reactions, such as anorexia and vomiting. There were no hypersensitivity reactions or chemotherapy-related mortality.

\section{Discussion}

It was reported that the median OS and PFS for patients with ENKTCL was only 7.8 and 5.8 months, respectively. According to the International Peripheral T-cell Lymphoma Project (22), ENKTCL exhibited the worst survival time among all peripheral T-cell lymphoma categories (19). The authors of that study concluded that radiotherapy was efficient for localized nasal disease, whereas extranasal disease appeared to be less amenable. For patients with advanced-stage nasal disease and those with extranasal disease, the survival was dismal $(<10 \%)$. In our study, we investigated the efficacy and safety of the GOLD regimen for patients with ENKTCL. The overall response rate (ORR) was 91\% (CR, 62\%; and PR, 29\%). The 1-, 2- and 3-year PFS and OS were 86, 64 and 57\% and 91, 80 and $74 \%$, respectively. The median follow-up for all the patients was 22 months and the median PFS and OS were not attained. Wang et al (8) reported that $67 \%$ of the patients were positive for $\mathrm{P}$-gp expression and the $\mathrm{CR}$ rate achieved in $\mathrm{P}$-gp-positive patients was significantly lower compared to that in P-gp-negative patients (20 vs. $60 \%$, respectively; $\mathrm{P}=0.045$ ) when treated with a cyclophosphamide, adriamycin, vincristine and prednisone (CHOP)-like regimen. As adriamycin and vincristine are substrates for P-gp, Yong et al (23) reported that ENKTCL patients treated with $\mathrm{CHOP}$ regimen followed by IFRT exhibited a CR rate of only $27 \%$. It is crucial to attain CR in patients with aggressive lymphoma. Several studies demonstrated that a CR with induction chemotherapy followed by radiotherapy may prolong survival in ENKTCL cases (24-26). Therefore, efforts have been focusing on designing novel chemotherapeutic regimens, effective in achieving a high CR rate. Nagafuji et al (12) first reported the case of a 60-year-old Japanese woman with stage IV ENKTCL who was treated with L-ASP and achieved a CR without disease progression for 18 months. Yamaguchi et al (27) conducted a phase II study of SMILE chemotherapy for ENKTCL and reported an ORR and CR of 79 and $45 \%$, respectively, with a 1-year survival rate of only $55 \%$. These results were inferior to those achieved with our regimen, possibly due to the fact that all the patients enrolled in the Yamaguchi et al study had relapsed or refractory disease. Of note, the SMILE regimen comprised the steroid dexamethasone, the multidrug resistance-unrelated agents methotrexate, ifosfamide and L-asparaginase, and etoposide, which was found to be efficient in vitro and in vivo against Epstein-Barr virus- associated lymphoproliferative disorders. Further analysis of that study found that the treatment-related toxicity was severe, with grade 4 neutropenia and infection in 92 and $61 \%$ of the cases, respectively (27). Considering the highly aggressive nature of ENKTCL, we designed a scheme of 14 days per cycle. We found that toxicity was alleviated when not using GEM on the eighth day. The safety of the GOLD regimen was also satisfactory. Grade 3/4 hematological toxicity was observed in only $16 \%$ of the cases $(9 / 55)$. Leucopenia and thrombocytopenia were the major manifestations and liver injury occurred in only 2 patients. All the adverse reactions were manageable with symptomatic treatment and there were no severe complications. In the 2013 ASCO Annual Meeting, Lin et al (24) reported the results of a phase II/III study on ENKTCL treated with CID-ATT, with a 1- and 3-year OS of 80.2 and $68 \%$ and a PFS of 74.9 and $60.5 \%$, respectively. The survival results of that study were similar to ours. The CID-ATT regimen includes administration of CHOP-B, ifosfamide + methotraxate + etoposide + dexamethasone (IMVD), and dexamethasone + cytarabine + cisplatin, in an alternating sequence, for a total of 6 courses ( 2 cycles). Further analysis in our study revealed significant differences in OS and PFS between early and advanced stages. Several previous studies had reported that Ann Arbor stage was a prognostic factor for OS and/or PFS $(24,28,29)$, although there remain controversies regarding the Ann Arbor staging system.

In conclusion, our study demonstrated that the GOLD regimen was highly effective and safe for patients with newly-diagnosed ENKTCL. However, the short follow-up and the retrospective design of the study may have affected the results to a certain extent. Therefore, a prospective trial should be conducted to further evaluate the efficacy and safety of the GOLD regimen for the treatment of ENKTCL patients.

\section{Acknowledgements}

This study was supported by grants from the National Natural Foundation of China (no. 81101797) and the Project of Henan Health Department (no. 2011020158).

\section{References}

1. Suzuki R, Takeuchi K, Ohshima K and Nakamura S: Extranodal NK/T-cell lymphoma: diagnosis and treatment cues. Hematol Oncol 26: 66-72, 2008.

2. Oshimi K: Progress in understanding and managing natural killer-cell malignancies. Br J Haematol 139: 532-544, 2007.

3. Liang Q, Ye ZY, Su ZL, et al: Clinicopathologic study of 963 cases of mature T-cell and natural killer/T-cell lymphoma with respect to 2008 WHO classification of lymphoid neoplasms. Chin J Pathol 39: 291-295, 2010 (In Chinese).

4. Li YX, Wang H, Jin J, et al: Radiotherapy alone with curative intent in patients with stage I extranodal nasal-type NK/T-cell lymphoma. Int J Radiat Oncol Biol Phys 82: 1809-1815, 2012

5. Li YX, Yao B, Jin J, Wang WH, et al: Radiotherapy as primary treatment for stage IE and IIE nasal natural killer/T-cell lymphoma. J Clin Oncol 24: 181-189, 2006.

6. Isobe $\mathrm{K}$, Uno T, Tamaru J, et al: Extranodal natural killer/T-cell lymphoma, nasal type: the significance of radiotherapeutic parameters. Cancer 106: 609-615, 2006.

7. Koom WS, Chung EJ, Yang WI, et al: Angiocentric T-cell and NK/T-cell lymphomas: radiotherapeutic viewpoints. Int J Radiat Oncol Biol Phys 59: 1127-1137, 2004.

8. Wang B, Li XQ, Ma X, Hong X, Lu H and Guo Y: Immunohistochemical expression and clinical significance of P-glycoprotein in previously untreated extranodal NK/T-cell lymphoma, nasal type. Am J Hematol 83: 795-799, 2008. 
9. Drénou B, Lamy T, Amiot L, et al: CD3- CD56 ${ }^{+}$non-Hodgkin's lymphomas with an aggressive behavior related to multidrug resistance. Blood 89: 2966-2974, 1997.

10. Jaccard A, Petit B, Girault S, et al: L-asparaginase-based treatment of 15 western patients with extranodal NK/T-cell lymphoma and leukemia and a review of the literature. Ann Oncol 20: 110-116, 2009.

11. Obama K, Tara M and Niina K: L-asparaginase-based induction therapy for advanced extranodal NK/T-cell lymphoma. Int J Hematol 78: 248-250, 2003.

12. Nagafuji K, Fujisaki T, Arima F and Ohshima K: L-asparaginase induced durable remission of relapsed nasal NK/T-cell lymphoma after autologous peripheral blood stem cell transplantation. Int J Hematol 74: 447-450, 2001.

13. Yong W, Zheng W, Zhu J, et al: L-asparaginase in the treatment of refractory and relapsed extranodal NK/T-cell lymphoma, nasal type. Ann Hematol 88: 647-652, 2009.

14. Ahn HK, Kim SJ, Hwang DW, Ko YH, Tang T, Lim ST and Kim WS: Gemcitabine alone and/or containing chemotherapy is efficient in refractory or relapsed NK/T-cell lymphoma. Invest New Drugs 31: 469-472, 2013.

15. Mounier N, El Gnaoui T, Tilly H, et al: Rituximab plus gemcitabine and oxaliplatin in patients with refractory/relapsed diffuse large B-cell lymphoma who are not candidates for high-dose therapy. A phase II Lymphoma Study Association trial. Haematologica 98: 1726-1731, 2013.

16. López A, Gutiérrez A, Palacios A, et al: GEMOX-R regimen is a highly effective salvage regimen in patients with refractory/relapsing diffuse large-cell lymphoma: a phase II study. Eur J Haematol 80: 127-132, 2008.

17. El Gnaoui T, Dupuis J, Belhadj K, et al: Rituximab, gemcitabine and oxaliplatin: an effective salvage regimen for patients with relapsed or refractory B-cell lymphoma not candidates for high-dose therapy. Ann Oncol 18: 1363-1368, 2007.

18. Nowak-Göttl U, Ahlke E, Fleischhack G, et al: Thromboembolic events in children with acute lymphoblastic leukemia (BFM protocols): prednisone versus dexamethasone administration. Blood 101: 2529-2533, 2003

19. Carbone PP, Kaplan HS, Musshoff K, Smithers DW and Tubiana M: Report of the Committee on Hodgkin's Disease Staging Classification. Cancer Res 31: 1860-1861, 1971.
20. Cheson BD, Horning SJ, Coiffier B, et al: Report of an international workshop to standardize response criteria for non-Hodgkin's lymphomas. NCI Sponsored International Working Group. J Clin Oncol 17: 1244, 1999

21. Trotti A, Colevas AD, Setser A, et al: CTCAE v3.0: development of a comprehensive grading system for the adverse effects of cancer treatment. Semin Radiat Oncol 13: 176-181, 2003.

22. Au WY, Weisenburger DD, Intragumtornchai $\mathrm{T}$, et al; International Peripheral T-Cell Lymphoma Project: Clinical differences between nasal and extranasal natural killer/T-cell lymphoma: a study of 136 cases from the International Peripheral T-Cell Lymphoma Project. Blood 113: 3931-3937, 2009.

23. Yong W, Zheng W and Zhang Y: Clinical characteristics and treatment of midline nasal and nasal type NK/T cell lymphoma. Zhonghua Yi Xue Za Zhi 81: 773-775, 2001 (In Chinese).

24. Lin N, Song Y, Zheng W, et al: A prospective phase II study of L-asparaginase- CHOP plus radiation in newly diagnosed extranodal NK/T-cell lymphoma, nasal type. J Hematol Oncol 6: 44, 2013.

25. Au WY, Lie AK, Liang R, et al: Autologous stem cell transplantation for nasal NK/T-cell lymphoma: a progress report on its value. Ann Oncol 14: 1673-1676, 2003.

26. Kim HJ, Bang SM, Lee J, et al: High-dose chemotherapy with autologous stem cell transplantation in extranodal NK/T-cell lymphoma: a retrospective comparison with non-transplantation cases. Bone Marrow Transplant 37: 819-824, 2006.

27. Yamaguchi M, Kwong YL, Kim WS, et al: Phase II study of SMILE chemotherapy for newly diagnosed stage IV, relapsed, or refractory extranodal natural killer (NK)/T-cell lymphoma, nasal type: the NK-Cell Tumor Study Group study. J Clin Oncol 29: 4410-4416, 2011.

28. Wu X, Li P, Zhao J, et al: A clinical study of 115 patients with extranodal natural killer/T-cell lymphoma, nasal type. Clin Oncol (R Coll Radiol) 20: 619-625, 2008.

29. Li YX, Fang H, Liu QF, et al: Clinical features and treatment outcome of nasal-type NK/T-cell lymphoma of Waldeyer ring. Blood 112: 3057-3064, 2008. 